\title{
Surgical Outcome and Ocular Complications of Evisceration and Intraocular Prosthesis Implantation in Dogs with End Stage Glaucoma: a Review of 20 Cases
}

\author{
Chung-Tien LIN $^{1) *}$, Chun-Kun HU' ${ }^{1)}$, Chen-Hsuan LIU ${ }^{1)}$ and Lih-Seng YEH ${ }^{1)}$ \\ ${ }^{1)}$ Department of Veterinary Medicine, College of Bio-Resources and Agriculture, National Taiwan University, Taipei, Taiwan
}

(Received 27 June 2006/Accepted 3 April 2007)

\begin{abstract}
Surgical outcome and complications following evisceration and implantation of intraocular silicone prosthesis (ISP) in 20 canine eyes with end-stage glaucoma were analyzed, including clinical signs, complications, cosmetic appearance, and owners' responses. The mean postoperative follow-up time was 312 days. Major short-term surgical complications were central corneal ulceration (6/20) and infections (3/20), while long-term complication was keratoconjunctivitis sicca (KCS) (2/20). All patient owners (100\%) showed satisfied with the surgical outcome and postoperative cosmetic effect including $85 \%$ of the owners gave an excellent or good rating of satisfaction. No more medication needed for long term control except the KCS cases. With careful case selection and postoperative care, evisceration with ISP implantation proves a good and safe surgical procedure with minimal complications for end stage glaucoma.
\end{abstract}

KEY WORDS: complications, end-stage glaucoma, intraocular silicone prosthesis (ISP).

J. Vet. Med. Sci. 69(8): 847-850, 2007

End stage glaucoma is clinically characterized by a persistent increased intraocular pressure (IOP) with buphthalmos, ocular pain, and refractory to medical treatment [4, 19]. This stage is commonly seen in dogs usually due to failure of early correct diagnosis or treatment, or rapid progression of the disease itself.

The treatment options for painful blind eyes caused by glaucoma include cyclocryotherapy or laser cryoablation, intraocular prosthesis implantation, and enucleation [2, 4, 5-8, 15, 17-19]. However, the improvement of end stage glaucoma by cryo- and laser-therapy is usually limited $[1,8$, $13,15,17]$. Owners usually hesitate to have the dog received enucleation surgery. The intraocular prosthesis implantation has become a popular alternative for end stage glaucoma to offer a painless and cosmetically acceptable eye after surgery $[6,7]$.

Here we present $15 \mathrm{dogs} / 20$ eyes with end stage glaucoma treated by evisceration and ISP implantation in Taiwan. The purpose of the study was to evaluate surgical complications, cosmetic appearance and owner responses following evisceration and ISP implantations in the dogs.

Medical records of 20 canine eyes with end-stage glaucoma treated with evisceration and ISP implantation at the Ophthalmology Clinic of the National Taiwan University Veterinary Hospital were analyzed. The dogs with intraocular infection or neoplasm were excluded for the procedure. The eyes with KCS or mild corneal ulcers were stabilized medically until substantial improvement or recovery prior to being considered to receive surgery. The eyes with pre-operatively stable and improving condition of KCS were also included for the surgery because postoperative

\footnotetext{
* Correspondenece to: Dr. Lin, C.-T., Department of Veterinary Medicine, College of Bio-Resources and Agriculture, National Taiwan University, No. 1, Section 4, Roosevelt Road, Taipei 106, Taiwan.
}

e-mail: ctlin@ntu.edu.tw size reduction of globe from buphthalmia and proper medical control of KCS usually work well for the cases with mild or moderate KCS cases. The cases of severe KCS were excluded for the surgery due to infection concern.

The operated eyes were prepared and draped as routine intraocular surgery. A $5-8 \mathrm{~mm}, 120^{\circ} \sim 150^{\circ}$, dorsal limbusbased conjunctival flap was created. The sclera was incised about $4-6 \mathrm{~mm}$ posterior and parallel to the limbus. The eye was eviscerated to remove intraocular contents using a lens loop. Following gently flushing intraocular space with saline, a sterile premeasured silicone implant (Veterinary Ophthalmic Supplies, Moscow, Idaho, U.S.A.) was carefully introduced into the fibrous tunics with a Carter sphere introducer. The size of prosthesis to be implanted was determined, by caliper, as $1 \mathrm{~mm}$ longer than the corneal diameter of the contralateral normal eye according to manufacturer's instruction. The scleral incision, bulbar conjunctiva, and Tenon's capsule were apposed with 6-0 absorbable polyglactin-910 suture (Vicryl ${ }^{\circledR}$, Ethicon, Inc., Somerville, U.S.A.) by simple continuous sutures.

The first recheck time was 2 nd day or 3rd day post-operatively, then every week for a month, and another 2 to 4 weeks for follow-up. The eyes were examined using a slit lamp biomicroscope. Ocular discharge, Schirmer's tear test, corneal fluorescein staining, conjunctival wound healing and globe size were routinely examined and at each followup. Successful surgery was defined as free of wound infection and no medication required following recovery. The owner's satisfaction rate for the surgical outcome was classified into four grades, namely excellent, good, acceptable, and bad base on the following four factors (criteria), including satisfactory post-operative cosmetic appearance, easy post-operative care (not labor intensive), satisfactory recovery and ocular comfort after surgery, and free of medication since recovery from surgery. The owner's satisfaction rate 
was classified as "excellent" if the owner's response met all of four criteria. The owner's satisfaction rate was classified as "good" if the owner's response met three criteria. The "acceptable" grade met two criteria and "bad" grade met one or no criteria. The data of owners' responses were gathered at visit and by telephone inquires.

The mean age of the patients was 8.2 years (range, 5 to 16 years). The gender distribution was 7 males and 8 females. Except for the mixed breed $(n=4)$, the Cocker spaniel (4) was the most common affected breed. Other breeds included Lhasa Apso (1), Shar-pie (1), Japanese Akita (1), Maltese (2), Shiba Inu (1), and miniature schnauzer (1). There were 9 cases affected in right eye, and 11 cases in left eye. The sphere diameter of ISP ranged from $14 \mathrm{~mm}$ to 18 $\mathrm{mm}$. The mean time of follow-up was 312 days, ranged from 30 to 573 days.

Corneal edema (19/20) and pigmentation (15/20) were the most common post-operative corneal appearance (Fig. 1 ), and they are considered an expected corneal reaction after prosthesis implantation [6]. The major post-operative complications that required monitoring or/and management were superficial to stromal corneal ulceration $(30 \%, 6 / 20)$ (Fig. 2), purulent discharge $(15 \%, 3 / 20)$, and KCS $(10 \%, 2 /$ 20). All of these complications were controlled well by appropriate management and had minimal effect on surgical outcome. The corneal ulcers occurred in 2 to 8 days after surgery and healed in 11 to 16 days post-operatively. There was only one case with central stromal corneal ulcer to be treated by 3rd eyelid flap surgery due to relatively larger corneal lesion and KCS. The ulcer healed and the flap was removed. No deep stromal corneal ulcers occurred in all cases. KCS occurred in 2 cases, one was diagnosed 2 weeks postoperatively, and the other one was diagnosed 4 months after surgery. In summary, the surgical complications were minimal and cosmetic effect was good (Fig. 3) in these cases. Table 1 shows a summary of the complications and outcome of the glaucoma patients following evisceration and intraocular prosthesis implantation.

Ninety \% (18/20) of operated eyes were comfortable and free of medication after recovery except for the two KCS cases $(2 / 20)$ on topical cyclosporine. Based on owner's response, an "excellent" rating of owners' satisfaction was received from $35 \%$ of cases, and a "good" rating from $50 \%$ of cases, resulting in an overall $85 \%(17 / 20)$ of surgical cases with either an excellent or good response for surgical outcome by the owners. There was no bad or unsatisfied rating from the case owners.

Only a few previous clinical papers describe surgical complications of the procedure to date. In previous case reports, the overall complication rate was ranged form $9 \%$ to $16 \%$. However, no detailed short-term complications were recorded in these reports. One of the most commonly reported complications of the procedure is ulcerative keratitis and most are healed with treatment. Other complications include wound dehiscence, panophthalmitis, excessive corneal neovascularization and scarring, and regrowth of intraocular neoplasm $[6,7,9,12]$.

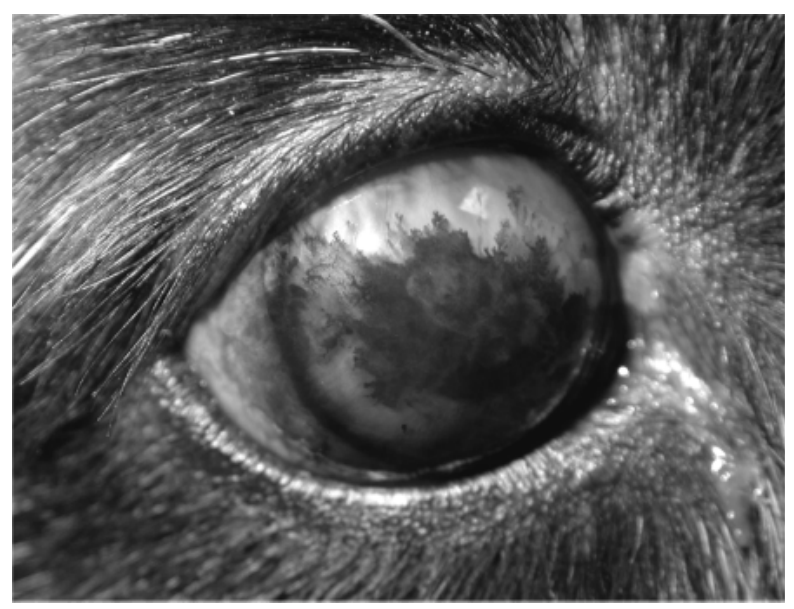

Fig. 1. An example of corneal edema and pigmentation after ISP implantation in a mongrel. The corneal edema or/and pigmentation were seen in most cases for short term after ISP implantation.

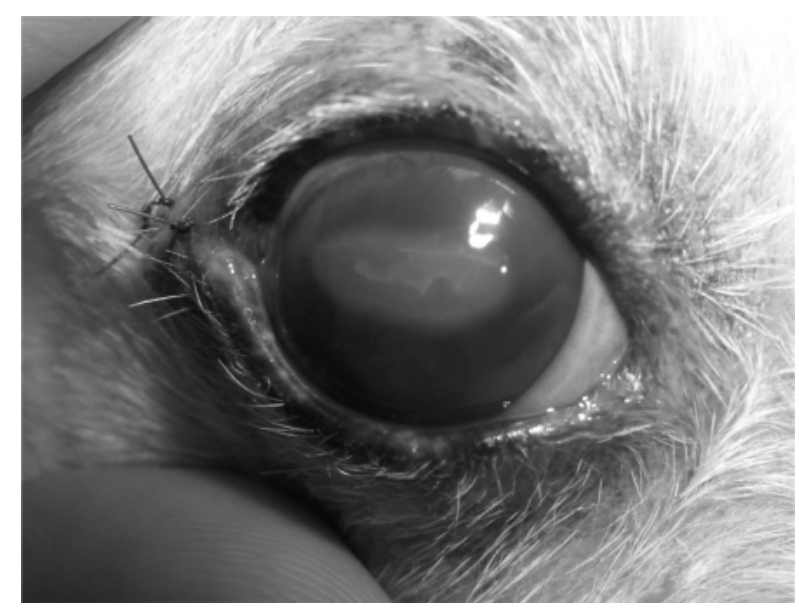

Fig. 2. A central stromal corneal ulcer in a Maltese after ISP surgery. A central stromal corneal ulcer was noticed in a Maltese 8 days after ISP surgery. KCS was diagnosed and the ulcer was healed following treatment.

In this study, the major post-operative complication was corneal ulceration. Six of 20 eyes were observed with superficial to stromal corneal ulcer in 2 to 8 days post-operatively. Although the incidence of postoperative corneal ulcers is higher than that in previous reports, all affected eyes remained mild lesions and lasted only for short term without signs of discomfort. Other previously reported complications were not observed in this study, such as implant extrusion and failed healing of scleral incision. The post-operative enucleation rate has been reported from 4.5 $8 \%$ in previous studies $[6,9]$, but no implant failure occurred in this study. The higher incidence of transient post-operative corneal ulcer in this study may attribute to that buphthalmic eyes (all cases) prone to develop corneal lesions. In the 6 eyes with post-operative corneal ulcers, $\mathrm{KCS}$ were diagnosed in 3 eyes, one KCS case was diag- 


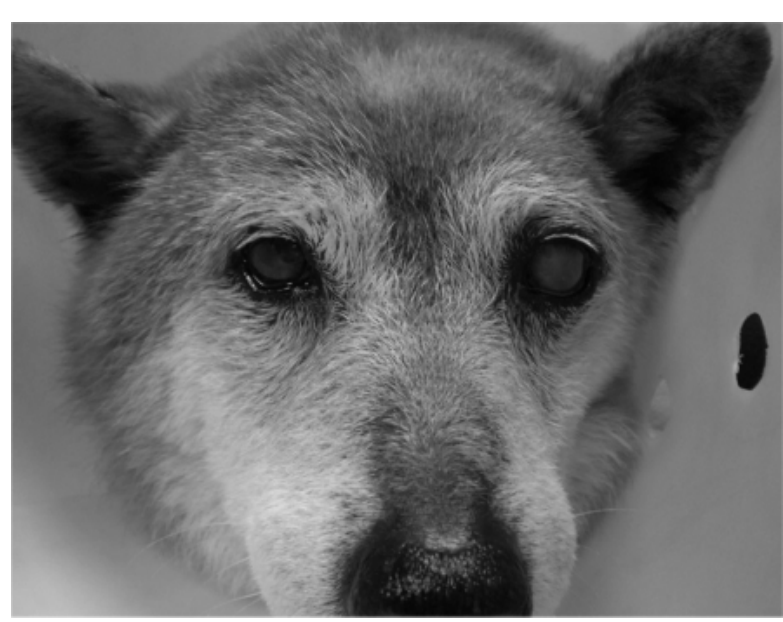

Fig. 3. Six months after bilateral ISP implantation surgery in a Shiba Inu with bilateral end stage glaucoma and lens luxation. The ocular appearance of a 16 year-old Shiba Inu with both eyes eviscerated and intraocular silicone implantation 6 months after surgery. Both eyes were comfortable and free of medication after recovery from surgery.

nosed before ulcer was developed and two KCS cases were diagnosed after ulcers were developed suggesting that those eyes are predisposed to keratoconjunctival lesions. In addition, the variation in incidence of corneal ulcer between the studies may be also explained by different sample sizes and postoperative follow-up time frame. We routinely have intense short term post-operative follow-up ophthalmic examinations, while other reports described an end-point long term outcome.

The development of post-operative ulcerative keratitis might be due to the following reasons: corneal exposure or damage during or after surgery [6]; the loss of uveal tract and aqueous humor that compromises corneal metabolism. In addition, disruption of innervation to cornea by large conjunctival and peri-limbal incision may contribute to a neurotrophic keratitis [10]. It is important to prevent corneal dryness during surgery and postoperatively [6], as drying or exposure of the cornea may contribute to transient ulcerative keratitis post-operatively. A post-operative temporary tarsorrhaphy may be helpful to prevent corneal exposure while the periocular tissues are swollen and eyes are buphthamic $[5,6,9,11]$.

Routine evaluation of tear production after surgery was performed in all cases during every follow-up visit in this study. KCS development as a complication of this procedure has not been reported. In this study we observed $2 / 20$ eyes with KCS diagnosed from 2 weeks and 4 months after surgery, respectively. Both cases were under very good control with topical cyclosporine. Interestingly we found that the KCS cases receiving topical $1 \%$ cyclosporine eye drop developed no or very little pigmentation on the cornea compared with other cases which had normal tear production. Corneal pigmentation is a non-specific response to corneal inflammation [19]. The topical cyclosporine may be helpful in reducing corneal pigment development by alleviating inflammatory irritation of the cornea after surgery. With the concern of infection and delayed wound healing, corticosteroid was not used in all cases as suggested $[3,19]$.

Corneal neovascularization, pigmentation and edema were common post-operative corneal reaction to the procedure. They may be caused by inflammatory cornea and damaged endothelium, but usually self-limited and become inactive in 4 to 6 weeks after surgery $[6,9,19]$.

Several types of intraocular prosthesis have been reported for use in dogs, including silicone, and methyl methacrylate (MMA) etc $[6,7,14,16]$. Intraocular silicone prosthesis (ISP) has the advantage of being inert, lightweight, non-

Table 1. The complications and outcome of the glaucoma patients following evisceration and intraocular prosthesis implantation in the dog

\begin{tabular}{|c|c|c|c|}
\hline $\begin{array}{l}\text { No. of } \\
\text { patient }\end{array}$ & Breed & Complications of the eyes & $\begin{array}{l}\text { Outcome of complication } \\
\text { management }\end{array}$ \\
\hline 1. & Cocker spaniel & Purulent ocular discharge & Infection was controlled after changing antibiotic \\
\hline 2. & Cocker spaniel & None & - \\
\hline 3. & Lhasa Apso & None & - \\
\hline 4. & Cocker spaniel & None & - \\
\hline 5. & Mongrel (bilateral) & None & - \\
\hline 6. & Cocker spaniel & Purulent ocular discharge & Infection was controlled after changing antibiotic \\
\hline 7. & Mongrel & Pre-op superficial corneal ulcer & Ulcer healed without additional treatment \\
\hline 8. & Maltese (Bilateral) & $\begin{array}{l}\text { Superficial corneal ulcer on } 3 \text { rd day, } \\
\text { and KCS occurred } 4 \text { months after surgery }\end{array}$ & $\begin{array}{l}\text { Ulcer healed without additional treatment, and KCS was } \\
\text { controlled by topical cyclosporine }\end{array}$ \\
\hline 9. & $\begin{array}{l}\text { Japanese Akita } \\
\text { (bilateral) }\end{array}$ & None & - \\
\hline 10. & Shiba Inu (bilateral) & Superficial corneal ulcer on 3rd day & Ulcer healed without additional treatment \\
\hline 11. & Maltese & $\begin{array}{l}\text { Central stromal corneal ulcer on } 8 \text { th day, } \\
\text { and KCS with purulent discharge occurred } \\
2 \text { weeks after surgery }\end{array}$ & $\begin{array}{l}\text { Ulcer healed after } 3 \text { rd eyelid flap surgery, and infection and } \\
\text { KCS were controlled after changing antibiotic and giving } \\
\text { topical cyclosporine }\end{array}$ \\
\hline 12. & Miniature schnauzer & $\begin{array}{l}\text { KCS diagnosed before surgery, and superficial } \\
\text { corneal ulcer on } 5 \text { th day }\end{array}$ & $\begin{array}{l}\text { KCS was controlled by cyclosporine, and ulcer healed } \\
\text { after swab debridement }\end{array}$ \\
\hline 13. & Mongrel & Superficial corneal ulcer on 2 nd day & Corneal ulcer healed without additional treatment \\
\hline 14. & Shar-pie (bilateral) & None & - \\
\hline 15. & Mongrel & Superficial corneal ulcer on 3rd day & Corneal ulcer healed without additional treatment \\
\hline
\end{tabular}


painful, nontoxic, non-antigenic, similar to the normal eye in appearance after surgery [5]. In previous studies, the sphere diameter size ranged from $16 \mathrm{~mm}$ to $20 \mathrm{~mm}$ in dogs $[6,9]$. We used $14 \mathrm{~mm}$ to $18 \mathrm{~mm}$ diameter size of ISP due to more small dog breeds in this study. Chronic low-grade conjunctivitis and secondary entropion have been reported by too small implanted prosthesis [6,9], yet a too large sphere may affect the tear distribution on the cornea. It is imperative to choose a proper sphere size to prevent these complications. The enlarged globe shrank to the size of the prosthesis over 3 to 6 weeks postoperatively in this study. During this period the cornea might vascularize and conjunctiva appeared hyperemia. These signs eventually resolved with time.

In summary, all surgeries were considered successful based on post-operative complications and owners' responses during follow-up in this study. Postoperative complications of the procedure were minimal and self-limited. All operated eyes were comfortable and free of medication after recovery except for the two KCS cases. With careful patient selection and good owner compliance, it is a superior surgical technique for dogs with end stage glaucoma.

\section{REFERENCES}

1. Brightman, A.H., Vestre, W.A., Helper, L.C. and Tomes, J.E. 1982. J. Am. Anim. Hosp. Assoc. 18: 319-322.

2. Cook, C.S. 1997. Vet. Clin. North. Am. Small. Anim. Pract. 27: 1109-1129.

3. Fowler, J.D. 1989. Semin. Vet. Med. Surg. (Small Anim). 4: 256-262.

4. Gelatt, K.N. 1999. pp. 526-528, 701-754. In: Veterinary Oph- thalmology. 3rd ed. Lippincott Williams \& Wilkins.

5. Gelatt, K.N. and Gelatt J.P. 2001. pp. 60-63. In: Small Animal Ophthalmic Surgery: Practical Technique for the Veterinarian. Butterworth-Heinemann.

6. Hamor, R.E., Whitley, R.D., McLaughlin, S.A., Lindley, D.M. and Albert, R.A. 1994. J. Am. Anim. Hosp. Assoc. 30: 66-69.

7. Hamor, R.E., Roberts, S.M. and Severin, G.A. 1993. J. Am Vet. Med. Assoc. 203: 701-706.

8. Hardman, C. and Stanley, R.G. 2001. Vet. Ophthalmol. 4: 209 215.

9. Koch, S.A. 1981. J. Am. Vet. Med. Assoc. 179: 883-885.

10. Lambiase, A., Bonini, S., Rama, P. and Olzi1, D. 2003. Eye 17: 989-995.

11. Martin, C.L. 2005. pp. 361-363. In: Ophthalmic Diseases in Veterinary Medicine. Manson Publishing Ltd.

12. McLaughlin, S.A., Ramsey, D.T., Lindley, D.M., Gilger, B.C., Gerding, P.A. and Whitley, R.D. 1995. J. Am. Vet. Med. Assoc. 207: 1441-1443.

13. Nasisse, M.P., Davidson, M.G., English, R.V., Jamieson, V., Harling, D.E. and Tate, L.P. 1990. J. Am. Vet. Med. Assoc. 197: 350-354.

14. Nasisse, M.P., Van Ee, R.T., Munger, R.J. and Davidson, M.G. 1988. J. Am. Vet. Med. Assoc. 192: 539-542.

15. O'Reilly, A., Hardman, C. and Stanley, R.G. 2003. Vet. Ophthalmol. 6: 113-119.

16. Pena, M.T., Luera, M. and Garcia, F.A. 1997. Vet. Rec. 140: 67-68.

17. Sapienza, J.S. and Van der Woerdt, A. 2005. Vet. Ophthalmol. 8: $121-127$.

18. Severin, G.A. 1996. pp. 464-470. In: Severin's Veterinary Ophthalmology Notes. 3rd ed., Veterinary Ophthalmology Notes.

19. Slatter, D. 2001. pp. 371-380. In: Fundamentals of Veterinary Ophthalmology. 3rd ed., W.B. Saunders Company. 